\title{
Double, Rydberg and Charge Transfer Excitations from Pairing Matrix Fluctuation and Particle-Particle Random Phase Approximation
}

Yang Yang, ${ }^{1}$ Helen van Aggelen, ${ }^{2,1}$ and Weitao Yang $^{3,}$ a)

1) Duke University, Department of Chemistry, Durham, NC 27r08, U.S.

${ }^{2)}$ Ghent University, Department of Inorganic and Physical Chemistry, 9000 Ghent, Belgium

3) Duke University, Department of Chemistry and Department of Physics, Durham, NC 27708 , U.S.

(Dated: 14 November 2013)

Double, Rydberg and charge transfer (CT) excitations have been great challenges for time-dependent density functional theory (TDDFT). Starting from an $(N \pm 2)$ electron single-determinant reference, we investigate excitations for the $N$-electron system through the pairing matrix fluctuation, which contains information on twoelectron addition/removal processes. We adopt the particle-particle random phase approximation (pp-RPA) and the particle-particle Tamm-Dancoff approximation (ppTDA) to approximate the pairing matrix fluctuation and then determine excitation energies by the differences of two-electron addition/removal energies. This approach captures all types of interesting excitations: single and double excitations are described accurately, Rydberg excitations are in good agreement with experimental data and CT excitations display correct 1/R dependence. Furthermore, the pp-RPA and the pp-TDA have a computational cost similar to TDDFT and consequently are promising for practical calculations.

a) Electronic mail: weitao.yang@duke.edu 


\section{INTRODUCTION}

The accurate description of excited states is an important and challenging topic. Information on excitation energies and oscillator strengths is necessary for explaining and predicting excitation spectra. Theoretical studies are also particularly helpful in determining the dynamics of electronically excited states. Many theoretical approaches have been developed for studying excited states. Full and multi-reference configuration interaction, methods with perturbative corrections to configuration interactions singles (including CIS-MP2 ${ }^{1}$ and CIS(D) ${ }^{2}$ ), complete active space (CAS) methods ${ }^{3}$, equation-of-motion and linear-response coupled cluster theories (EOM-CC and LR-CC) ${ }^{4,5}$ are generally accurate but computationally expensive. For large molecules, only a few single-determinant reference approaches are more applicable. These approaches include configuration interaction singles $(\mathrm{CIS})^{6}$, time-dependent Hartree-Fock (TDHF) ${ }^{7}$ and time-dependent density functional theory $(\text { TDDFT })^{8-11}$. CIS and TDHF are also known as the Tamm-Dancoff approximation (TDA) and the random phase approximation (RPA), respectively. They have the same single-determinant reference - the Hartree-Fock ground state, which is a poor first-order approximation with no correlation and overestimated HOMO-LUMO gaps. At the same time, excitation operators of these two methods are limited to particle-hole excitations. Therefore, CIS and TDHF tend to overestimate excitation energies and are only capable of capturing single excitations. Furthermore, TDHF often suffers from instabilities for triplet states ${ }^{12,13}$, which makes it much less used. TDDFT is based on Kohn-Sham reference states and is more accurate in predicting excitation energies than CIS and TDHF. However, within the adiabatic approximation, in which the exchange-correlation kernel is frequency-independent, TDDFT also can only capture single excitations ${ }^{14,15}$. Because of their incorrect long-range

behavior $^{12}$, approximate exchange-correlation kernels also have difficulties describing Rydberg excitations. Moreover, because of their delocalization/self-interaction error, TDDFT greatly underestimates charge transfer (CT) excitations and has no 1/R Coulomb interaction character, with $\mathrm{R}$ the separation distance ${ }^{11,12,16,17}$. Therefore, an efficient method that can accurately deal with single, double, Rydberg and CT excitations all together is particularly valuable and highly desirable.

Besides above traditional methods that use $N$-electron ground states as starting points, many non- $N$-electron-ground-state reference methods have also been developed to inves- 
tigate excitation problems. These methods are mostly in the framework of EOM-CC ${ }^{18}$. For example, the spin-flip (SF-) EOM-CC ${ }^{19,20}$ method uses an $N$-electron high-spin triplet reference, the ionization-potential/electron-attachment (IP/EA-) EOM-CC ${ }^{21,22}$ use $(N \pm 1)$ electron ground states, the double ionization-potential/electron-attachment (DIP/DEA-) EOM-CC ${ }^{23,24}$ use $(N \pm 2)$-electron ground states and the triple ionization-potential/electronattachment (TIP/TEA-) EOM-CC ${ }^{25}$ can use $(N \pm 3)$-electron ground states. Such EOMCC methods involving electron number changes have roots in Fock-Space coupled cluster (FSCC) theory ${ }^{26-33}$ and similarity transformed EOM-CC ${ }^{34,35}$. A similarity among these non- $N$-electron-ground-state reference methods is that the $N$-electron ground state and excited states are constructed with the same procedure, therefore, these methods are believed have balanced treatment between the ground state and excited states. Furthermore, the change of references provides much more choices in solving excitation problems. However, these well-developed variants EOM-CC are also computationally expensive. Fortunately, SF-TDDFT ${ }^{36}$, which only uses a single-determinant high-spin triplet reference provides a computationally more efficient alternative to SF-EOM-CC and has found its practical use in the prediction of double excitations ${ }^{37}$. However, there are currently no single-determinant alternatives to EOM-CC methods based on ground states with different electron number. In this paper, we present such a single-determinant alternative to (DIP/DEA-) EOM-CC.

This approach we propose in this paper adopts $(N \pm 2)$-electron single-determinant references to investigate excitation problems. This approach is based on the pairing matrix fluctuation, which contains information on two-electron addition and removal processes as well as the ground state correlation energy ${ }^{38,39}$. The pairing matrix fluctuation has been applied to the investigation of Auger Spectroscopy ${ }^{40,41}$. However, it has never been used to investigate neutral excitations. We start from single-determinant $(N \pm 2)$-electron references and from the pairing matrix fluctuation for these references, we obtain information on transitions both to the ground state and to the excited states of the $N$-electron system. With this information excitation energies and oscillator strengths can be determined. Although the exact pairing matrix fluctuation, which should give exact excitation energies, is unknown, the particle-particle random phase approximation (pp-RPA) and the particle-particle TammDancoff approximation (pp-TDA) provide useful first-order approximations. Unlike CIS, TDHF and TDDFT approaches, which essentially adopt the particle-hole channel of interactions to solve excitation problems, this new approach adopts the particle-particle channel. 
In the following sections, we will show its good performance in describing double, Rydberg, CT and single excitations.

\section{THEORY}

The pairing matrix is defined as $\kappa_{i j}(t)=\left\langle\Psi_{0}^{N}\left|a_{H_{i}}(t) a_{H_{j}}(t)\right| \Psi_{0}^{N}\right\rangle^{42}$, in which $\Psi_{0}^{N}$ is the $N$-electron ground state and $a_{H_{j}}(t)$ is the annihilation operator in Heisenberg picture on orbital $i$ at time $t$. In the absence of any pairing field, normal atomic and molecular systems stay in eigenstates of the particle number operator and the pairing matrix is always zero. However, when a perturbing pairing field $\hat{F}\left(t^{\prime}\right)=\sum_{k l} f_{k l}\left(t^{\prime}\right) a_{H_{l}}^{\dagger}\left(t^{\prime}\right) a_{H_{k}}^{\dagger}\left(t^{\prime}\right) \theta\left(t^{\prime}\right)$ is tuned on after time 0 , the pairing matrix will respond to this field and give non-zero results. $\overline{\mathbf{K}}\left(t-t^{\prime}\right)$ describes this kind of response, and it is also equal to the pairing matrix fluctuation. ${ }^{38}$ The matrix elements of $\overline{\mathbf{K}}\left(t-t^{\prime}\right)$ are

$$
\bar{K}_{i j k l}\left(t-t^{\prime}\right)=-\frac{i}{\hbar} \theta\left(t-t^{\prime}\right)\left\langle\Psi_{0}^{N}\left|\left[a_{H_{i}}(t) a_{H_{j}}(t), a_{H_{l}}^{\dagger}\left(t^{\prime}\right) a_{H_{k}}^{\dagger}\left(t^{\prime}\right)\right]\right| \Psi_{0}^{N}\right\rangle
$$

We can Fourier transform $\overline{\mathbf{K}}\left(t-t^{\prime}\right)$ to the energy domain and the elements of the corresponding $\overline{\mathbf{K}}(E)$ takes the following form.

$$
\begin{aligned}
\bar{K}(E)_{i j k l} & =\sum_{n} \frac{\left\langle\Psi_{0}^{N}\left|a_{i} a_{j}\right| \Psi_{n}^{N+2}\right\rangle\left\langle\Psi_{n}^{N+2}\left|a_{l}^{+} a_{k}^{+}\right| \Psi_{0}^{N}\right\rangle}{E-\omega_{n}^{N+2}+i \eta} \\
& -\sum_{n} \frac{\left\langle\Psi_{0}^{N}\left|a_{l}^{+} a_{k}^{+}\right| \Psi_{n}^{N-2}\right\rangle\left\langle\Psi_{n}^{N-2}\left|a_{i} a_{j}\right| \Psi_{0}^{N}\right\rangle}{E-\omega_{n}^{N-2}+i \eta}
\end{aligned}
$$

$\bar{K}(E)_{i j k l}$ has poles at the two-electron addition and removal energies $\omega_{n}^{N+2}$ and $\omega_{n}^{N-2}$. If we compute for an $(N-2)$-electron system instead of an $N$-electron system, the two-electron addition energies $\omega_{n}^{N}$ describe the transitions from the $(N-2)$-electron ground state $\left|\Psi_{0}^{N-2}\right\rangle$ to the $N$-electron states $\left|\Psi_{n}^{N}\right\rangle$. The differences between the transition energies that involve the ground and the $n$-th excited $N$-electron state can then be characterized as $N$-electron excitation energies:

$$
E_{n}^{N}-E_{0}^{N}=\left(E_{n}^{N}-E_{0}^{N-2}\right)-\left(E_{0}^{N}-E_{0}^{N-2}\right)=\omega_{n}^{N}-\omega_{0}^{N}
$$

Therefore, exact $N$-electron excitation energies can be obtained from the exact pairing matrix fluctuation $\overline{\mathbf{K}}(E)$ of the $(N-2)$-electron system. 
Although the exact pairing matrix fluctuation in general is difficult to compute, the pp-RPA ${ }^{38,42}$ provides a way of approximating this fluctuation

$$
\overline{\mathbf{K}}=\overline{\mathbf{K}}^{0}+\overline{\mathbf{K}}^{0} \mathbf{V} \overline{\mathbf{K}}
$$

where $\overline{\mathbf{K}}^{0}$ is the pairing matrix fluctuation for a non-interacting reference and $\mathbf{V}$ is the Coulomb interaction. This approximation can be applied to both HF references and DFT references and can be further formulated into an eigenvalue problem ${ }^{38}$

$$
\left[\begin{array}{ll}
\mathbf{A} & \mathbf{B} \\
\mathbf{B}^{\dagger} & \mathbf{C}
\end{array}\right]\left[\begin{array}{l}
\mathbf{X}^{n} \\
\mathbf{Y}^{n}
\end{array}\right]=\omega_{n}\left[\begin{array}{cc}
\mathbf{I} & \mathbf{0} \\
\mathbf{0} & -\mathbf{I}
\end{array}\right]\left[\begin{array}{l}
\mathbf{X}^{n} \\
\mathbf{Y}^{n}
\end{array}\right]
$$

with

$$
\begin{aligned}
& A_{a b, c d}=\delta_{a c} \delta_{b d}\left(\epsilon_{a}+\epsilon_{b}\right)+\langle a b \| c d\rangle \\
& B_{a b, k l}=\langle a b \| k l\rangle \\
& C_{i j, k l}=-\delta_{i k} \delta_{j l}\left(\epsilon_{i}+\epsilon_{j}\right)+\langle i j \| k l\rangle
\end{aligned}
$$

where $a, b, c, d$ are particle indices and $i, j, k, l$ are hole indices with restrictions that $a>$ $b, c>d, i>j$ and $k>l$. Eigenvectors dominated by $\mathbf{X}$ components describe the transition amplitudes of two-electron addition processes with $X_{a b}^{n}=\left\langle\Psi_{0}^{N}\left|a_{a} a_{b}\right| \Psi_{n}^{N+2}\right\rangle$ and $Y_{i j}^{n}=\left\langle\Psi_{0}^{N}\left|a_{i} a_{j}\right| \Psi_{n}^{N+2}\right\rangle$. Eigenvalues for these eigenvectors are two-electron addition energies $\omega_{n}^{N+2}=E_{n}^{N+2}-E_{0}^{N}$. Similarly, eigenvectors dominated by $\mathbf{Y}$ components describe the transition amplitudes of two-electron removal processes with $X_{a b}^{n}=\left\langle\Psi_{0}^{N}\left|a_{b}^{+} a_{a}^{+}\right| \Psi_{n}^{N-2}\right\rangle$ and $Y_{i j}^{n}=\left\langle\Psi_{0}^{N}\left|a_{j}^{+} a_{i}^{+}\right| \Psi_{n}^{N-2}\right\rangle$. The corresponding eigenvalues are two-electron removal energies $\omega_{n}^{N-2}=E_{0}^{N}-E_{n}^{N-2}$.

A Tamm-Dancoff approximation can be applied to the pp-RPA. Setting $\mathbf{B}$ to be $\mathbf{0}$, the eigenvalue problem breaks into two separate blocks. The pp-TDA part

$$
\mathbf{A} \mathbf{X}^{n}=\omega_{n} \mathbf{X}^{n}
$$

has only $\mathbf{X}$ components and describes the two-electron addition process, while the hh-TDA part

$$
\mathbf{C Y}^{n}=-\omega_{n} \mathbf{Y}^{n}
$$

has only $\mathbf{Y}$ components and describes the two-electron removal process.

The pp-RPA, pp-TDA and hh-TDA can also be derived from the equation of motion $(\mathrm{EOM})^{43,44}$. In general, given an $N$-electron ground state $\left|\Psi_{0}^{N}\right\rangle$, all other $M$-electron states 
$\left|\Psi_{n}^{M}\right\rangle$ can be regarded as excited states. The exact excitation operators can be expressed as

$$
O^{\dagger}=\left|\Psi_{n}^{M}\right\rangle\left\langle\Psi_{0}^{N}\right|
$$

The equation of motion is

$$
\left[\hat{H}, O^{\dagger}\right]=\left(E_{n}^{M}-E_{0}^{N}\right) O^{\dagger}
$$

and applying a probing de-excitation $\delta O$ gives

$$
\left[\delta O,\left[\hat{H}, O^{\dagger}\right]\right]=\left(E_{n}^{M}-E_{0}^{N}\right)\left[\delta O, O^{\dagger}\right]
$$

Note that here we derived the key equation (Eq.11) as an exact operator equation without dependence on references. However, we still need to project the operator equation on a reference to carry out matrix element evaluations. In practice, the projection is often onto a HF reference, which is an approximation to the exact ground state $\left|\Psi_{0}^{N}\right\rangle$. We also need to expand and then truncate the excitation operator. The expansion space usually can be spanned by all the possible combinations of creation and annihilation operators defined with the $\mathrm{HF}$ reference orbitals. If we simply restrict $O^{\dagger}$ to $2 \mathrm{p}$-addition excitations and $\delta O$ to 2 p-removal de-excitations, the EOM will give the pp-TDA equation. Similarly, if $O^{\dagger}$ only includes $2 \mathrm{~h}$-removal excitations and $\delta O$ only includes $2 \mathrm{~h}$-addition de-excitations, the EOM will recover the hh-TDA equation. The pp-RPA can also be recovered by allowing $O^{\dagger}$ to include both $2 \mathrm{p}$-addition and $2 \mathrm{~h}$-addition excitations and $\delta O$ to include both $2 \mathrm{p}$-removal and 2h-removal de-excitations.

However, as an approximated ground state, the references need not be restricted to an $(N-2)$-electron HF determinant. DFT references, multi-determinant references, or even non-optimized artificial references can be adopted. In this work, in addition to optimized single-determinant $(N-2)$-electron references, we consider an $N$-electron system in which the two highest occupied orbitals are treated as virtual orbitals, denoted as the HF* reference (Detailed equations for $\mathrm{HF}^{*}$ can be found in Section IB of Ref. ${ }^{45}$ ). Essentially, this $\mathrm{HF}^{*}$ reference is a non-optimized single-determinant $(N-2)$-electron reference and it is similar in spirit to spin-flip methods, which use high-spin triplet $N$-electron references. As to DFT references, if we argue in the framework of EOM, we should use HF-like orbital energies based on DFT orbitals. However, in the rest of the paper, when we use DFT references, we simply use the (generalized) Kohn-Sham orbitals and eigenvalues in Eq.6, and we will justify this approximation in a future paper. 


\section{COMPUTATIONAL DETAILS}

Our implementations and calculations were all based on the QM4D package ${ }^{46}$. For BH and $\mathrm{CH}^{+}$, geometries were taken from Ref. ${ }^{47}$. The remaining geometries were taken from the G2/97 set, which were optimized using MP2(full)/6-31G*48. Rydberg excitations were investigated with atomic species as well as the $\mathrm{N}_{2}$ and $\mathrm{CO}$ molecule. Calculations on atoms

used extensive even-tempered basis sets ${ }^{49,50}$, with exponents satisfying $\alpha_{i}=\alpha_{1} \beta^{i-1}$. Each basis contains 20s, 17p and 15d functions, with the smallest exponent $\alpha_{1}=0.00097656$ and the rest growing by a factor of $\beta=2$. Calculations on $\mathrm{N}_{2}$ and $\mathrm{CO}$ used a triply augmented cc-pVTZ ${ }^{51}$. CT excitations were investigated with $\mathrm{He}_{2}$ and $\mathrm{C}_{2} \mathrm{H}_{4} \cdot \mathrm{C}_{2} \mathrm{~F}_{4}$ model systems. Since only the qualitative $1 / \mathrm{R}$ behavior was of present interest, we used the simple cc-pVDZ and $6-31 \mathrm{G}^{*}$, respectively. For $\mathrm{He}_{2}$, a point charge was positioned far from the molecule to break the symmetry. For $\mathrm{BH}$ and $\mathrm{CH}^{+}$, cc-pVQZ basis sets were used. For polyenes, we used an augmented cc-pVDZ basis for $\mathrm{C}$ and a cc-pVDZ basis for $\mathrm{H}$.

\section{RESULTS}

\section{A. Double excitations}

The main strength of the pp-RPA is that it captures double excitations accurately (Table I). For Beryllium, with $\mathrm{HF}$ and $\mathrm{HF}^{*}$ references, the errors for double excitations are within $0.1 \mathrm{eV}$. With the B3LYP reference, the errors are slightly larger, but also within $1 \mathrm{eV}$. In this few-electron atomic system, the pp-RPA and the pp-TDA hardly show any differences $(<0.01 \mathrm{eV})$. For BH, the pp-RPA also captures double excitations. Compared to EOM$\mathrm{CCSD}(\mathrm{T})$ results, $\mathrm{HF}$ and $\mathrm{HF}^{*}$ references show errors of about $0.2 \mathrm{eV}$ and B3LYP references show errors of about $0.1 \mathrm{eV}$. The excitations with double excitation character in all-trans1,3-butadiene and 1,3,5-hexatriene are also captured. Compared to experimental data or accurate ab initio methods, HF and B3LYP references give relatively accurate results, while the $\mathrm{HF}^{*}$ reference overestimates the excitation energies by about $1.5-2 \mathrm{eV}$. The pp-RPA and the pp-TDA show some differences $(\approx 0.1 \mathrm{eV})$ in these larger systems, but the differences are still too small to conclude which approximation is better. 
TABLE I. Lowest double excitations or excitations with double excitation character (in eV)

\begin{tabular}{lccccc}
\hline \hline Term & Standard & RPA-HF & TDA-HF & RPA-B3LYP & RPA-HF* \\
\hline Be & & & & & \\
${ }^{1} \mathrm{D}$ & 7.05 & 7.06 & 7.06 & 7.97 & 7.06 \\
${ }^{3} \mathrm{P}$ & 7.40 & 7.45 & 7.45 & & 7.45 \\
$\mathrm{BH}$ & & & & 5.12 & 5.53 \\
${ }^{3} \Sigma$ & 5.04 & 5.51 & 5.48 & 5.98 & 6.18 \\
${ }^{1} \Delta$ & 6.06 & 6.15 & 6.12 & 7.05 & 7.22 \\
${ }^{1} \Sigma$ & 7.10 & 7.11 & & 7.93 \\
${ }^{\mathrm{butadiene}}$ & & & & 6.47 & \\
${ }^{1} \mathrm{~A}_{\mathrm{g}}$ & 6.55 & 5.93 & 5.83 & & 7.46 \\
hexatriene & & 5.43 & 5.34 & 5.01 & \\
${ }^{1} \mathrm{~A}_{\mathrm{g}}$ & 5.21 & & & & \\
\hline \hline
\end{tabular}

${ }^{a}$ In all the tables, RPA is short for pp-RPA and TDA is short for pp-TDA. Standard values are experimental data for $\mathrm{Be}^{52}$ and hexatriene ${ }^{53}, \mathrm{EOM}-\mathrm{CCSD}(\mathrm{T}) / \mathrm{cc}-\mathrm{pVQZ}$ results for $\mathrm{BH}^{47}, \mathrm{MR}-\mathrm{CISD}(\mathrm{Q})$ results for butadiene ${ }^{54}$.

\section{B. Rydberg excitations}

The pp-RPA describes Rydberg excitations well (Table II). With HF or HF* references, the pp-RPA describes Rydberg excitation energies within $0.03 \mathrm{eV}$ for Beryllium. For openshell Lithium, in spite of some spin contamination, results are also in good agreement with experimental data, with errors smaller than $0.1 \mathrm{eV}$. For molecules such as $\mathrm{N}_{2}$, errors are about $1.2 \mathrm{eV}$, which is better than TDLDA results $(\approx 2 \mathrm{eV})^{50}$. Calculations with the B3LYP reference overestimate Rydberg excitations and do not perform as well as with the HF reference (Table II, IV, V, VIII and IX in Ref. ${ }^{45}$ ).

\section{CT excitations}

The pp-RPA is capable of describing CT excitations (Figure 1 for $\mathrm{C}_{2} \mathrm{H}_{4} \cdot \mathrm{C}_{2} \mathrm{~F}_{4}$ and Figure 2 for $\left.\mathrm{He}_{2}\right)$. The computed CT excitations show exact $1 / \mathrm{R}$ dependence, with $\mathrm{R}$ the separation 
TABLE II. Rydberg excitations (in eV)

\begin{tabular}{|c|c|c|c|c|c|}
\hline Transition & Term & Standard & RPA-HF & TDA-HF & RPA-HF* \\
\hline \multicolumn{6}{|l|}{$\mathrm{Be}$} \\
\hline $2 \mathrm{~s} \rightarrow 6 \mathrm{~s}$ & ${ }^{3} \mathrm{~S}$ & 8.82 & 8.79 & 8.79 & 8.79 \\
\hline $2 \mathrm{~s} \rightarrow 6 \mathrm{~s}$ & ${ }^{1} \mathrm{~S}$ & 8.84 & 8.81 & 8.81 & 8.81 \\
\hline $2 \mathrm{~s} \rightarrow 6 \mathrm{p}$ & ${ }^{3} \mathrm{P}$ & 8.89 & 8.87 & 8.87 & 8.87 \\
\hline $2 \mathrm{~s} \rightarrow 6 \mathrm{p}$ & ${ }^{1} \mathrm{P}$ & 8.90 & 8.87 & 8.87 & 8.87 \\
\hline $2 \mathrm{~s} \rightarrow 6 \mathrm{~d}$ & ${ }^{3} \mathrm{D}$ & 8.93 & 8.91 & 8.91 & 8.91 \\
\hline $2 \mathrm{~s} \rightarrow 6 \mathrm{~d}$ & ${ }^{1} \mathrm{D}$ & 8.96 & 8.95 & 8.95 & 8.95 \\
\hline \multicolumn{6}{|l|}{$\mathrm{Li}$} \\
\hline $2 \mathrm{~s} \rightarrow 6 \mathrm{~s}$ & ${ }^{2} \mathrm{~S}$ & 4.96 & 4.97 & 4.97 & - \\
\hline $2 \mathrm{~s} \rightarrow 6 \mathrm{p}$ & ${ }^{2} \mathrm{P}$ & 5.01 & 5.05 & 5.05 & - \\
\hline $2 \mathrm{~s} \rightarrow 6 \mathrm{~d}$ & ${ }^{2} \mathrm{D}$ & 5.01 & 5.03 & 5.03 & - \\
\hline \multicolumn{6}{|l|}{$\mathrm{N}_{2}$} \\
\hline$\sigma_{\mathrm{g}} \rightarrow 3 \mathrm{~s} \sigma_{\mathrm{g}}$ & ${ }^{3} \Sigma_{\mathrm{g}}^{+}$ & 12.0 & 10.97 & 10.39 & - \\
\hline$\sigma_{\mathrm{g}} \rightarrow 3 \mathrm{~s} \sigma_{\mathrm{g}}$ & ${ }^{1} \Sigma_{\mathrm{g}}^{+}$ & 12.2 & 11.07 & 10.69 & - \\
\hline$\sigma_{\mathrm{g}} \rightarrow 3 \mathrm{p} \pi_{\mathrm{u}}$ & ${ }^{1} \Pi_{\mathrm{u}}$ & 12.90 & 11.62 & 11.26 & - \\
\hline$\sigma_{\mathrm{g}} \rightarrow 3 \mathrm{p} \sigma_{\mathrm{u}}$ & ${ }^{1} \Sigma_{\mathrm{u}}^{+}$ & 12.98 & 11.63 & 11.29 & - \\
\hline
\end{tabular}

a Standard values are all experimental data ${ }^{52,55}$. Li has no hole-hole pairs and consequently RPA and TDA calculations are the same. Since our current implementation on the pp-RPA-HF* is only for closed-shell systems with non-degenerate HOMO orbitals, so no data are available for Li nor for $\mathrm{N}_{2}$ because its HF HOMO orbitals are degenerate $\pi$, which is incorrect.

distance. Other non-CT excitations remain constant with respect to $\mathrm{R}$. In these two systems, because HF and DFT calculations on the $(N-2)$-electron references give nearly degenerate and delocalized HOMO and LUMO orbitals and are hard to converge, we cannot perform further pp-RPA calculations. Fortunately, with the HF* reference, the HOMO orbital for the N-electron system is non-degenerate and localized and can be treated as unoccupied. Through pairing matrix fluctuation, two electrons can be added either both to the same molecule, thus describing non-CT excitations, or to a different molecule each, thus describing 


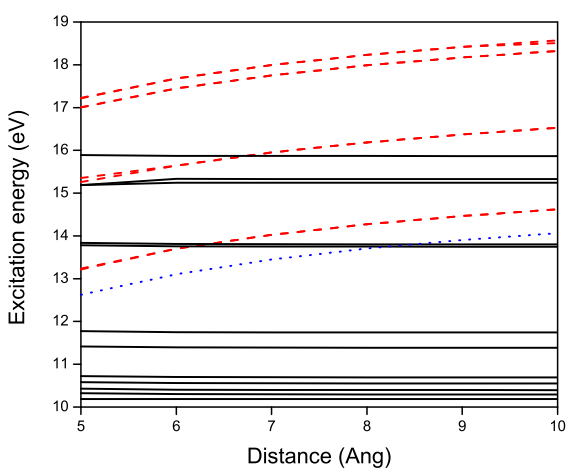

FIG. 1. CT and non-CT excitations calculated with pp-RPA-HF* for $\mathrm{C}_{2} \mathrm{H}_{4} \cdot \mathrm{C}_{2} \mathrm{~F}_{4}$ (The lowest non-CT excitation is not shown). Non-CT excitations are denoted with black solid lines and they show a constant behavior. CT excitations are denoted with red dashed lines and they increase when distance increases. This increasing behavior is "parallel" to the dotted blue line, which is a shifted $1 / \mathrm{R}$ reference.

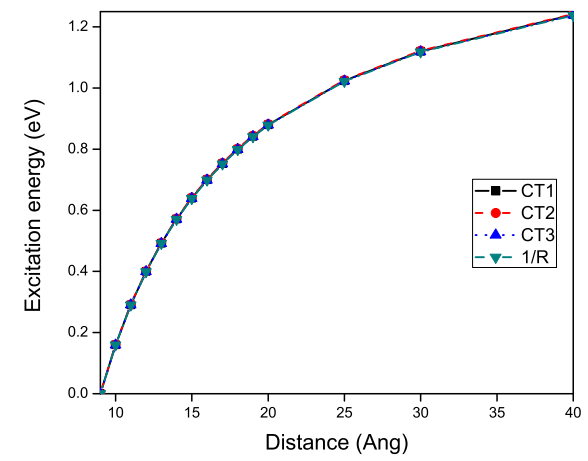

FIG. 2. CT excitations calculated with pp-RPA-HF* for $\mathrm{He}_{2}$. Excitation energies for the three CT states at 9 Åare all set to zero. The $1 / \mathrm{R}$ reference is also shifted to zero at $9 \AA$. All these three CT excitations show an exact $1 / \mathrm{R}$ behavior.

CT excitations.

\section{Single excitations}

The pp-RPA describes single excitations well. A small selection of single excitations is shown in Table III. For the two-electron system He, the pp-RPA reduces to the pp-TDA and they have a complete expansion of excitation operators. Consequently, they are exact for two-electron systems. For other cases, such as $\mathrm{C}, \mathrm{N}_{2}$ and $\mathrm{CO}$ (Table IV, VIII and IX in 
TABLE III. Single excitations (in eV)

\begin{tabular}{lccccc}
\hline \hline Term & Standard & RPA-HF & RPA-B3LYP & RPA-HF* & TDDFT \\
\hline $\mathrm{Mg}$ & & & & & \\
${ }^{3} \mathrm{P}$ & 2.71 & 2.57 & 3.43 & 2.58 & 2.61 \\
${ }^{1} \mathrm{P}$ & 4.53 & 4.27 & 5.68 & 4.28 & 4.25 \\
${ }^{3} \mathrm{~S}$ & 5.11 & 4.99 & 6.96 & 5.00 & 5.06 \\
${ }^{1} \mathrm{~S}$ & 5.39 & 5.27 & 7.21 & 5.28 & 5.45 \\
${ }_{\mathrm{CH}}^{+}$ & & & & & \\
${ }^{3} \Pi$ & 1.15 & 1.72 & 1.16 & 3.31 & -1.24 \\
${ }^{1} \Pi$ & 3.60 & 3.18 & 3.50 & 2.83 \\
$\mathrm{CO}$ & 3.07 & & & & \\
${ }^{3} \Pi$ & 6.32 & 5.59 & 5.84 & 6.44 & 5.96 \\
${ }^{1} \Pi$ & 8.51 & 7.77 & 7.99 & 9.32 & 8.42 \\
\hline \hline
\end{tabular}

${ }^{a}$ Standard values are experimental data for $\mathrm{Be}^{52}$ and $\mathrm{CO}^{55}$, EOM-CCSD(T)/cc-pVQZ results for $\mathrm{CH}^{+47}$. TDDFT results come from TD-B3LYP calculations for $\mathrm{Mg}$ and $\mathrm{CH}^{+47}$ and TD-LDA calculations for $\mathrm{CO}^{50}$

Ref. ${ }^{45}$ ), we notice some missing excitations from orbitals below HOMO and these missing excitations will be discussed in section IV E. For all other single excitations that we captured, the pp-RPA and the pp-TDA give good results comparable with TDDFT (with B3LYP or LDA).

The good quality of pp-RPA and pp-TDA excitation energies benefits from the same level of correlated descriptions for both the ground and the excited states, based on a common $(N-2)$-electron reference. This is an advantage of all the non-ground-state-reference approaches. Therefore, not only the pp-RPA can capture some excitations that are missing or particularly challenging for TDDFT, but also it describes single excitations as well as TDDFT. 
TABLE IV. Oscillator strengths (in A.U.) for $\mathrm{H}_{2}$ calculated with the pp-TDA-HF* and TDHF

\begin{tabular}{ccc}
\hline \hline Transition & pp-TDA-HF* & TDHF \\
\hline $\mathrm{HOMO} \rightarrow \mathrm{HOMO}+1$ & 0.28 & 0.29 \\
$\mathrm{HOMO} \rightarrow \mathrm{HOMO}+2$ & 0 & 0 \\
$\mathrm{HOMO} \rightarrow \mathrm{HOMO}+3$ & 0.35 & 0.35 \\
$\mathrm{HOMO} \rightarrow \mathrm{HOMO}+4$ & 0 & 0 \\
$\mathrm{HOMO} \rightarrow \mathrm{HOMO}+5$ & 0.03 & 0.04 \\
$\mathrm{HOMO} \rightarrow \mathrm{HOMO}+6$ & 0.82 & 0.83 \\
$\mathrm{HOMO} \rightarrow \mathrm{HOMO}+7$ & 0.83 & 0.83 \\
$\mathrm{HOMO} \rightarrow \mathrm{HOMO}+8$ & 0 & 0 \\
\hline \hline
\end{tabular}

${ }^{a}$ Both calculations are performed using the $6-31++\mathrm{G}^{* *}$ basis set. The pp-TDA shows good agreement with TDHF.

\section{E. Missing excitations}

Some excitations from orbitals below HOMO (Table IV, VIII and IX in Ref. ${ }^{45}$ ) are missing. This is a limitation of our current implementation, in which the orbitals below the HOMO are all occupied in all the references. Therefore only excitations from the HOMO orbital are present in the excitation spectrum. However, because of the flexibility of choosing reference configurations, a $\mathrm{HF}^{*}$ reference with lower-energy orbitals unoccupied or a spin-flip $(N-2)$-electron reference or a multi-determinant reference should retrieve these excitations.

\section{F. Oscillator strengths}

Oscillator strengths can be calculated with the pp-TDA. As the eigenvectors $\mathbf{X}$ describe transition amplitudes between the $(N-2)$-electron ground state and all the $N$-electron states, we can calculate all the $N$-electron wavefunctions and transition dipoles between any two $N$-electron states (Detailed derivation can be found in Sec. ID in Ref. ${ }^{45}$ ). A test on $\mathrm{H}_{2}$ agrees well with TDHF results (Table IV). 
TABLE V. Excitations for S atom (in eV)

\begin{tabular}{ccccccc}
\hline \hline Configuration & Term & Expt & RPA-HF & TDA-HF & RPA-B3LYP & TDA-B3LYP \\
\hline $\mathrm{S}$ & & & & & & \\
$3 \mathrm{~s}^{2} 3 \mathrm{p}^{4}$ & $1 \mathrm{D}$ & 1.15 & 1.07 & 1.13 & 1.04 & 1.11 \\
$3 \mathrm{~s}^{2} 3 \mathrm{p}^{4}$ & $1 \mathrm{~S}$ & 2.75 & 1.93 & 2.26 & 1.71 & 2.08 \\
$3 \mathrm{~s} 3 \mathrm{p}^{5}$ & $3 \mathrm{P}^{\circ}$ & 8.93 & 13.15 & 13.08 & 10.17 & 10.08 \\
$\mathrm{O}$ & & & & & & \\
$2 \mathrm{~s}^{2} 2 \mathrm{p}^{4}$ & $1 \mathrm{D}$ & 1.97 & 1.49 & 1.59 & 1.43 & 2.56 \\
$2 \mathrm{~s}^{2} 2 \mathrm{p}^{4}$ & $1 \mathrm{~S}$ & 4.19 & 2.70 & 3.11 & 2.39 & 2.83 \\
$2 \mathrm{~s} 2 \mathrm{p}^{5}$ & $3 \mathrm{P}^{\circ}$ & 15.66 & 19.28 & 19.16 & 14.87 & 14.71 \\
\hline \hline
\end{tabular}

${ }^{\text {a }}$ Experimental values are from Ref. ${ }^{52}$. All calculations start with $(N+2)$-electron references and use the cc-pVQZ basis set.

\section{G. Excitations from $(N+2)$-electron reference systems}

We also performed preliminary tests on the two electron-removal part with $(N+2)$ electron references (Tabel V). On the whole, the results has relatively large errors. However, from these two limited cases, it seems that the HF reference is better for HOMO excitations, while the B3LYP reference is better for lower-orbital excitations.

\section{H. Cost evaluation}

Our current implementation is based on direct matrix diagonalization on Eq.5, which gives all the eigenvalues and eigenvectors. The cost of computing all $O\left(N^{2}\right)$ eigenvalues is roughly $O\left(N^{6}\right)$. However, this is not the most efficient way, since only a small fraction of excitation spectrum is usually of interest, the states of interest can be computed with the Davidson algorithm $^{56}$ in the pp-TDA to achieve the $O\left(N^{4}\right)$ scaling. A similar implementation should also be possible for the pp-RPA. Therefore, these approaches should have a computational cost similar to TDDFT. They are probably the first known approaches that describe single, double, Rydberg and CT excitations well with only $O\left(N^{4}\right)$ cost, and may have a promising future in practical calculations. 


\section{CONCLUSIONS}

In this paper, we proposed an approach that uses pairing matrix fluctuations to solve excitation problems. It can be viewed as a single-reference counterpart of the DIP/DEA-EOM$\mathrm{CC}$ method which uses coupled-cluster references. It is also the particle-particle channel counterpart of CIS, TDHF and TDDFT, which essentially adopt the particle-hole channel. Furthermore, it also has similar philosophy to SF-TDDFT, which is a non-ground-state single-determinant starting point. The pp-RPA, pp-TDA and hh-TDA provide reasonable approximations to the pairing matrix fluctuation and give rise to good descriptions of double, Rydberg, CT and single excitations. Although the current implementation has restrictions only to the HOMO excitations, the problem can be tackled by adopting more flexible references. These methods should have computational cost similar to TDDFT after implementation of Davidson algorithm and thus may be promising for practical calculations.

\section{ACKNOWLEDGMENTS}

Support from FWO-Flanders (Scientific Research Fund Flanders) (H.v.A), the Office of Naval Research (N00014-09- 0576) and the National Science Foundation (CHE-09-11119) (W.Y.) is appreciated. We also greatly thank Degao Peng and Dr. Stephan N. Steinmann for helpful discussions.

\section{REFERENCES}

${ }^{1}$ J. B. Foresman, M. Head-Gordon, J. A. Pople, and M. J. Frisch, The Journal of Physical Chemistry 96, 135 (1992).

${ }^{2}$ M. Head-Gordon, R. J. Rico, M. Oumi, and T. J. Lee, Chemical Physics Letters 219, 21 (1994).

${ }^{3}$ B. O. Roos, The Complete Active Space Self-Consistent Field Method and its Applications in Electronic Structure Calculations, pages 399-445, John Wiley \& Sons, Inc., 2007.

${ }^{4}$ K. Emrich, Nuclear Physics A 351, 379 (1981).

${ }^{5}$ H. J. Monkhorst, International Journal of Quantum Chemistry 12, 421 (1977).

${ }^{6}$ J. E. D. Bene, R. Ditchfield, and J. A. Pople, The Journal of Chemical Physics 55, 2236 (1971). 
${ }^{7}$ A. D. Mclachlan and M. A. Ball, Rev. Mod. Phys. 36, 844 (1964).

${ }^{8}$ E. Runge and E. K. U. Gross, Phys. Rev. Lett. 52, 997 (1984).

${ }^{9}$ M. E. Casida, Time-dependent density functional response theory of molecular systems: Theory, computational methods, and functionals, in Recent Developments and Applications of Modern Density Functional Theory, edited by J. Seminario, volume 4 of Theoretical and Computational Chemistry, pages 391 - 439, Elsevier, 1996.

${ }^{10}$ M. Petersilka, U. J. Gossmann, and E. K. U. Gross, Phys. Rev. Lett. 76, 1212 (1996).

${ }^{11}$ C. Ullrich, Time-Dependent Density-Functional Theory: Concepts and Applications, Oxford Graduate Texts, OUP Oxford, 2012.

${ }^{12}$ A. Dreuw and M. Head-Gordon, Chemical Reviews 105, 4009 (2005).

${ }^{13}$ J. Cížek and J. Paldus, The Journal of Chemical Physics 47, 3976 (1967).

${ }^{14}$ D. J. Tozer and N. C. Handy, Phys. Chem. Chem. Phys. 2, 2117 (2000).

${ }^{15}$ R. J. Cave, F. Zhang, N. T. Maitra, and K. Burke, Chemical Physics Letters 389, 39 (2004).

${ }^{16}$ D. J. Tozer, R. D. Amos, N. C. Handy, B. O. Roos, and L. Serrano-Andres, Molecular Physics 97, 859 (1999).

${ }^{17}$ A. Dreuw, J. L. Weisman, and M. Head-Gordon, The Journal of Chemical Physics 119, 2943 (2003).

${ }^{18}$ A. I. Krylov, Annual Review of Physical Chemistry 59, 433 (2008), PMID: 18173379.

${ }^{19}$ A. I. Krylov, Chemical Physics Letters 338, 375 (2001).

${ }^{20}$ A. I. Krylov, Accounts of Chemical Research 39, 83 (2006).

${ }^{21}$ J. F. Stanton and J. Gauss, The Journal of Chemical Physics 101, 8938 (1994).

${ }^{22}$ M. Nooijen and R. J. Bartlett, The Journal of Chemical Physics 102, 3629 (1995).

${ }^{23}$ K. W. Sattelmeyer, H. F. S. III, and J. F. Stanton, Chemical Physics Letters 378, 42 (2003).

${ }^{24}$ J. Shen and P. Piecuch, The Journal of Chemical Physics 138, 194102 (2013).

${ }^{25}$ M. Musiał, M. Olszówka, D. I. Lyakh, and R. J. Bartlett, The Journal of Chemical Physics 137, $174102(2012)$.

${ }^{26}$ I. Lindgren, International Journal of Quantum Chemistry 14, 33 (1978).

${ }^{27}$ M. A. Haque and D. Mukherjee, The Journal of Chemical Physics 80, 5058 (1984).

${ }^{28}$ L. Z. Stolarczyk and H. J. Monkhorst, Phys. Rev. A 32, 725 (1985).

${ }^{29}$ L. Z. Stolarczyk and H. J. Monkhorst, Phys. Rev. A 32, 743 (1985). 
${ }^{30}$ L. Z. Stolarczyk and H. J. Monkhorst, Phys. Rev. A 37, 1908 (1988).

${ }^{31}$ L. Z. Stolarczyk and H. J. Monkhorst, Phys. Rev. A 37, 1926 (1988).

${ }^{32}$ D. Mukherjee and S. Pal, Advances in Quantum Chemistry 20, 291 (1989).

${ }^{33}$ U. Kaldor, Theoretica chimica acta 80, 427 (1991).

${ }^{34}$ M. Nooijen and R. J. Bartlett, The Journal of Chemical Physics 106, 6441 (1997).

${ }^{35}$ M. Nooijen and R. J. Bartlett, The Journal of Chemical Physics 106, 6449 (1997).

${ }^{36}$ Y. Shao, M. Head-Gordon, and A. I. Krylov, The Journal of Chemical Physics 118, 4807 (2003).

${ }^{37}$ Z. Rinkevicius, O. Vahtras, and H. A. gren, The Journal of Chemical Physics 133, 114104 (2010).

${ }^{38}$ H. van Aggelen, Y. Yang, and W. Yang, Phys. Rev. A 88, 030501 (2013).

${ }^{39}$ Y. Yang, H. van Aggelen, S. N. Steinmann, D. Peng, and W. Yang, The Journal of Chemical Physics 139, 174110 (2013).

${ }^{40}$ C.-M. Liegener, Chemical Physics Letters 90, 188 (1982).

${ }^{41}$ S. Taioli, S. Simonucci, L. Calliari, and M. Dapor, Physics Reports 493, 237 (2010).

${ }^{42} \mathrm{~J}$. Blaizot and G. Ripka, Quantum theory of finite systems, Cambridge, MA, 1986.

${ }^{43}$ D. J. Rowe, Rev. Mod. Phys. 40, 153 (1968).

${ }^{44}$ P. Ring and P. Schuck, The Nuclear Many-Body Problem, Physics and astronomy online library, Springer, 2004.

${ }^{45}$ Supplemental materials, available at...

${ }^{46} \mathrm{An}$ in-house program for $\mathrm{qm} / \mathrm{mm}$ simulations (http://www.qm4d.info).

${ }^{47}$ D. Zhang, S. N. Steinmann, and W. Yang, The Journal of Chemical Physics 139, 154109 (2013).

${ }^{48}$ L. A. Curtiss, K. Raghavachari, P. C. Redfern, and J. A. Pople, The Journal of Chemical Physics 106, 1063 (1997).

${ }^{49}$ T. Helgaker, P. Jørgensen, and J. Olsen, Molecular electronic-structure theory, Wiley, 2000.

${ }^{50}$ Q. Wu, A. J. Cohen, and W. Yang, Molecular Physics 103, 711 (2005).

${ }^{51}$ D. E. Woon and J. Thom H. Dunning, The Journal of Chemical Physics 100, 2975 (1994).

${ }^{52}$ A. E. Kramida, Y. Ralchenko, J. Reader, and N. A. Team, NIST Atomic Spectra Database (version 5.0), 2012. 
${ }^{53}$ T. Fujii, A. Kamata, M. Shimizu, Y. Adachi, and S. Maeda, Chemical Physics Letters 115, 369 (1985).

${ }^{54}$ M. Dallos and H. Lischka, Theoretical Chemistry Accounts 112, 16 (2004).

${ }^{55}$ D. J. Tozer and N. C. Handy, The Journal of Chemical Physics 109, 10180 (1998).

${ }^{56}$ E. R. Davidson, Journal of Computational Physics 17, 87 (1975). 